\title{
Discriminability of differences in line slope and in line arrangement as a function of mask delay*
}

\author{
JACOB BECK and BRUCE AMBLER \\ University of Oregon, Eugene, Oregon 97403
}

Position uncertainty was used to guarantee that an S's attention was distributed equally over a visual display. Diminishing discriminability through a masking flash, which controlled the time available to attend selectively to the individual figures in the display, increased the relative discriminability of figures differing in their line slope as compared to figures differing in their line arrangement. Diminishing discriminability through decreasing the clarity of a visual trace by reducing exposure duration did not increase the relative discriminability of figures differing in their line slopes. It is suggested that the discriminability of differences in line slope and in line arrangement varies with the distribution of attention. The results are interpreted as supporting the hypothesis that, under distributed attention in peripheral vision, the visual system is more sensitive to differences in line slope than to differences in line arrangement.

Studies of the effectiveness of figural and textural variables in producing perceptual grouping have led to the conclusion that similarity grouping occurs most strongly on the basis of simple properties of stimuli (Beck, 1967, 1972; Olson \& Attneave, 1970; Julesz, 1962). Research, for example, has shown that differences in the slopes of the component lines of figures produce strong grouping, while differences in the arrangement of the component lines of figures produce weak or little grouping. The fact that similarity grouping does not occur strongly on the basis of a relational variable, such as the arrangement of lines, suggests that the processes that underlie grouping are based on discriminative responses to stimulus differences. This suggestion is further supported by the fact that the rated similarity in appearance of figures has little relationship to the grouping that occurs (Beck, 1966).

Beck (1972) proposed that primary similarity grouping involves segregating a pattern into groups on the basis of stimulus differences that are easily discriminable when attention is not concentrated but is distributed over a wide field. He reported data which showed that the effectiveness of differences in line slope and in line arrangement in producing similarity grouping corresponded to their peripheral discriminability in a patterned visual field in which an $S$ did not know where to attend. In contrast,

*This research was supported by National Science Foundation Grant GB-24884 and ARPA Contract F44620 67C-0099. These data were reported at the meetings of the Psychonomic Society, St. Louis, Missouri, November 1971 . the relative discriminability of differences in line slope and in line arrangement, when presented in an empty visual field, did not correspond to their effectiveness in producing similarity grouping. This difference in the discriminability of figural differences in a patterned and in an empty visual field was interpreted as resulting from a narrowing or focusing of an S's attention. In an empty visual field, the brightness difference between a figure and background is able to direct an $S$ where to attend. Recent investigations have shown that attentional focusing may affect form discrimination (Eriksen \& Collins, 1969; Eriksen \& Rohrbaugh, 1970).

The present experiments were designed to provide further information on the relative discriminability of differences in line slope and in line arrangement under concentrated and distributed attention. The basic experimental manipulation was the interval between the offset of a stimulus array of letters and the onset of poststimulus masks in the areas that the letters had occupied. Position uncertainty was used to guarantee that an S's attention was distributed equally over the display. If a masking field immediately follows a briefly flashed stimulus array, an $S$ is not able to attend to the individual figures in the array. Accordingly, one would expect that, with short masking delays, discriminability would be based upon distributed attention. If a difference in line slope is more discriminable than a difference in line arrangement when attention is distributed over a wide field, then figures differing in the slopes of their lines would be more discriminable than figures differing in the arrangement of their lines. At the other extreme, when no masking field is presented, discriminability would also reflect the information obtainable by selectively attending to individual figures of the stimulus array in the fading visual trace. The relative discriminability of figures differing in the arrangement of their lines under these conditions may be expected to improve.

\section{EXPERIMENT 1}

Subjects

Eight volunteers with normal vision without correction served as paid Ss. They were naive as to the purpose of the experiment.

\section{Apparatus and Stimuli}

The experiment was programmed on a PDP-15 computer, and the stimuli were displayed on a Hewlett-Packard 1300A oscilloscope with a Pll phosphor. The face of the ocsilloscope was masked to present a circular field 8 in. in diam. The stimulus arrays consisted of six letters arranged around the circumference of an imaginary circle, 5 in. in diam, at 0 , $60,120,180,240$, and 300 deg. Five of the letters were upright Ts. The position of the sixth letter, which was determined randomly, was a disparate letter and was either an $L$ or a $T$, tilted $33 \mathrm{deg}$ from the vertical. The $L$ figure, which differs in the arrangement of its component lines from an upright $T$, was chosen because in previous experiments, of those figures having vertical and horizontal lines, the $L$ was the most discriminable from an upright $\mathbf{T}$. A 33-deg tilted $\mathbf{T}$ was chosen because, in pilot studies, a $\mathrm{T}$ tilted $33 \mathrm{deg}$ from the vertical was found to be less or equally discriminable from an upright $T$ as was an $\mathrm{L}$ when no masking field followed the presentation of a stimulus array.

Each of the two lines making up a figure was composed of 7 dots. Since there was a common dot at the conjunction of two lines, each figure contained 13 dots. The length of the lines of dots making up an upright $T$, an $\mathrm{L}$, and a tilted $\mathrm{T}$ was about $.36 \mathrm{in}$. The masks consisted of 10 by 10 matrices of dots in the areas that the letters had occupied. Each mask completely covered a letter and was about .5 in. square. Foveal inspection indicated that the letters became indistinguishable against the masking pattern as soon as the mask appeared. The individual dots making up the letters and masks were not discriminable in peripheral vision and appeared as lines and surfaces of fine texture. The letters and masks were presented at a low level of brightness, and the brightness ratio of the masks to the letters was approximately $4: 1$.

Each $\mathrm{S}$ was dark adapted for $10 \mathrm{~min}$ 


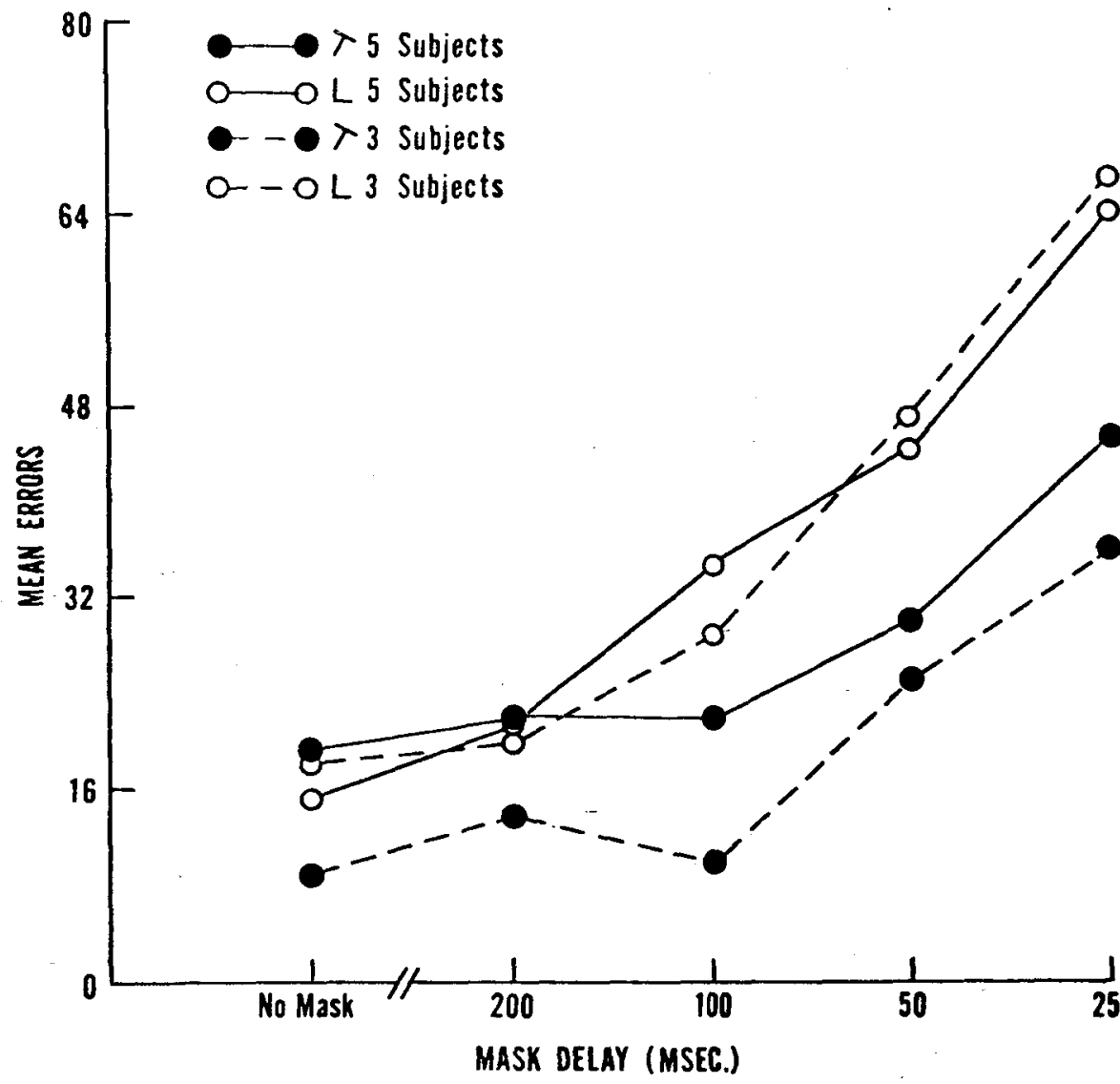

Fig. 1. Mean errors for a tilted $T$ and an $L$ as a function of mask delay in Experiment 1. The points connected by solid lines are based on 960 trials (5 Ss $x$ 192 trials), and the points connected by broken lines are based on 576 trials (3 Ss $\times 192$ trials).

in a dimly illuminated room before beginning the experiment. A 100-W bulb, shielded by a reflector run at $35 \mathrm{~V}$, was placed in the corner of the room, $6 \mathrm{ft}$ from a $S$ and directed at the ceiling. The oscilloscope was completely enclosed in a compartment. A chinrest, which assured a constant binocular viewing distance of approximately $9.5 \mathrm{in}$., was placed within the compartment. The compartment in which the oscilloscope and chinrest were located was completely dark except for the illumination supplied by the display. The stimulus arrays were viewed binocularly at eye level, with fixation in the center of the field. The Ss were instructed to respond by pressing one of two keys to indicate whether a stimulus array consisted of all upright Ts or contained a disparate figure such as an $\mathrm{L}$ or a tilted $\mathrm{T}$. Five different masking delays were used: no mask, $200,100,50$, and 25 msec.

\section{Procedure}

A within-Ss design, in which Ss were run for 5 consecutive days, was employed. Four experimental days followed a practice day. The practice response. After an $\mathrm{S}$ responded, the computer waited either 1.2 or $1.8 \mathrm{sec}$ before presenting the next stimulus. These delays were chosen randomly.

Each $S$ was presented with 480 trials on each of the 4 experimental days. Five blocks of 48 trials (one block for each of the five masking conditions) were presented with a tilted $T$ as the disparate figure and five blocks of 48 trials with the $L$ as the disparate figure. In a block of 48 trials, 24 stimulus arrays having only upright Ts were mixed randomly with 24 stimulus arrays having a disparate figure. The position of the disparate figure varied randomly from trial to trial, so that Ss never knew where the disparate figure would be presented. The 48 trials in a block were broken into subblocks of 12 trials. The Ss were given a 10-sec rest between each subblock of 12 trials and a 2 -min rest between blocks of 48 trials. The Ss were instructed to fixate a central dot, .06 in. square, and were told that this would give them the best chance of making the most correct responses.

A different irregular order of each of the five masking delays was prepared for each $\mathrm{S}$. This order was used on the first 2 experimental days. On the 3rd and 4 th experimental days, a different irregular order was prepared in which the masking delays that were presented at the beginning of the experimental session on Days 1 and 2 were presented toward the end of the experimental session on Days 3 and 4 . The order of presenting a tilted $T$ or an $L$ as the disparate figure was alternated within each of the experimental conditions over days.

familiarized an $\mathbf{S}$ with the task was used to determine an exposure duration, which gave an error rate of approximately $10 \%$ when no masking field was presented. On each of the experimental days, an $\mathrm{S}$ was given practice trials with the no-mask condition before beginning the actual experiment. If the error rate on the practice trials was similar to that previously obtained, the experiment was begun. If the error rate on these practice trials differed from that obtained on the initial practice day, a slightly different exposure duration was used during the experimental session. For four Ss, the same exposure duration was used on all 4 experimental days; the exposure duration was changed slightly on either 1 or 2 of the experimental days for the other four Ss. The mean exposure time for the eight $\mathrm{Ss}$ was $52.8 \mathrm{msec}$ and ranged from 45 to $60 \mathrm{msec}$. After each stimulus presentation, an $S$ was given 2 sec in which to respond. If an $S$ did not respond within this time, the computer recorded an error and presented the next stimulus. The masking field was terminated by an S's

\section{Results}

Figure 1 presents the mean errors for each of the five masking delays. The data points are based on 192 trials per S. 1 The eight Ss have been separated into two groups. For five Ss, the discriminability of the tilted $T$ was worse than the discriminability of the $L$ when no masking field was presented. The mean errors for these five Ss are shown by solid lines. For three $\mathrm{Ss}$, the discriminability of a even when no masking field was presented. The mean errors for these three Ss are shown by the broken lines. It is clear from Fig. 1 that the difference between the two groups is due to the better discriminability of the tilted $T$ for three of the Ss. The discriminability of the $L$ was similar for all Ss.

The overall trends of the two groups are similar. The data indicate that the mean errors for an $L$ increased more than the mean errors for a tilted $T$ with short masking delays. As the interval between the offset of the tilted $T$ was better than that of an $L$ 
stimulus array and the onset of a masking field was decreased, the discriminability of a tilted $T$ improved relative to the discriminability of an $L$. There is a crossover at $200 \mathrm{msec}$ of the $\mathrm{L}$ and the tilted $\mathrm{T}$ mean error curves for the five $S$ s in which the discriminability of a tilted $T$ was initially worse.

Figure 2 presents the combined results for the eight Ss. An analysis of variance revealed a significant effect due to whether the disparate figure was an $L$ or a tilted $T[F(1,7)=12.22$, $p<.02]$ and the delay of the masking field $[F(4,28)=39.64, p<.01]$. The analysis of variance also indicated that the Figure by Masking Delay interaction was significant $[\mathrm{F}(4,28)=6.93, \mathrm{p}<.01]$. Statistical tests of the differences between the mean errors for the $\mathrm{L}$ and the tilted $\mathrm{T}$ at $100 \mathrm{msec}(t=5.83), 50 \mathrm{msec}$ $(t=3.50)$, and 25 msec $(t=3.55)$ were significant $(p<.01)$. Though an a posteriori comparison raises problems about the significance level, it should be pointed out that all eight Ss made more errors with a 100-msec mask delay than with no mask when an $L$ was the disparate figure (mean error increase was 17.1 with a SD of the difference scores of 20 ), while five Ss made more errors and three Ss fewer errors when a tilted $T$ was the disparate figure (mean error increase was 2.1 with a SD of the difference scores of 10.4). The results were similar when the data was analyzed in terms of $d^{\prime}$. The proportions of hits and false alarms (excluding the trials on which Ss failed to respond) gave d's of $2.74,2.52,1.98,1.52$, and .78 when an $L$ was the disparate figure and $d$ 's of $2.98,2.60,2.68,2.08$, and 1.61 when a tilted $T$ was the disparate figure for the no-mask, 200-, 100-, 50-, and 25-msec masking delays. At masking delays of $100 \mathrm{msec}$ and less, the $d^{\prime}$ values show that the sensitivity to a tilted $T$ is greater than the sensitivity to an $\mathrm{L}$.

The finding that the discriminability of a tilted $\mathrm{T}$ is better than that of an $\mathrm{L}$ with short masking delays is consistent with the hypothesis that the differences in the slopes of the lines of a tilted $\mathrm{T}$ are more discriminable when attention is distributed over a wide field than is the difference in the arrangement of the lines of an $L$. With longer masking delays, attention may be focused or concentrated on individual figures of the stimulus array, and the relative discriminability of an $\mathrm{L}$ improves.

\section{EXPERIMENT 2}

Experiment 2 was designed to compare the relative discriminability of a tilted $T$ and an $L$ in patterns of upright Ts when discriminability was

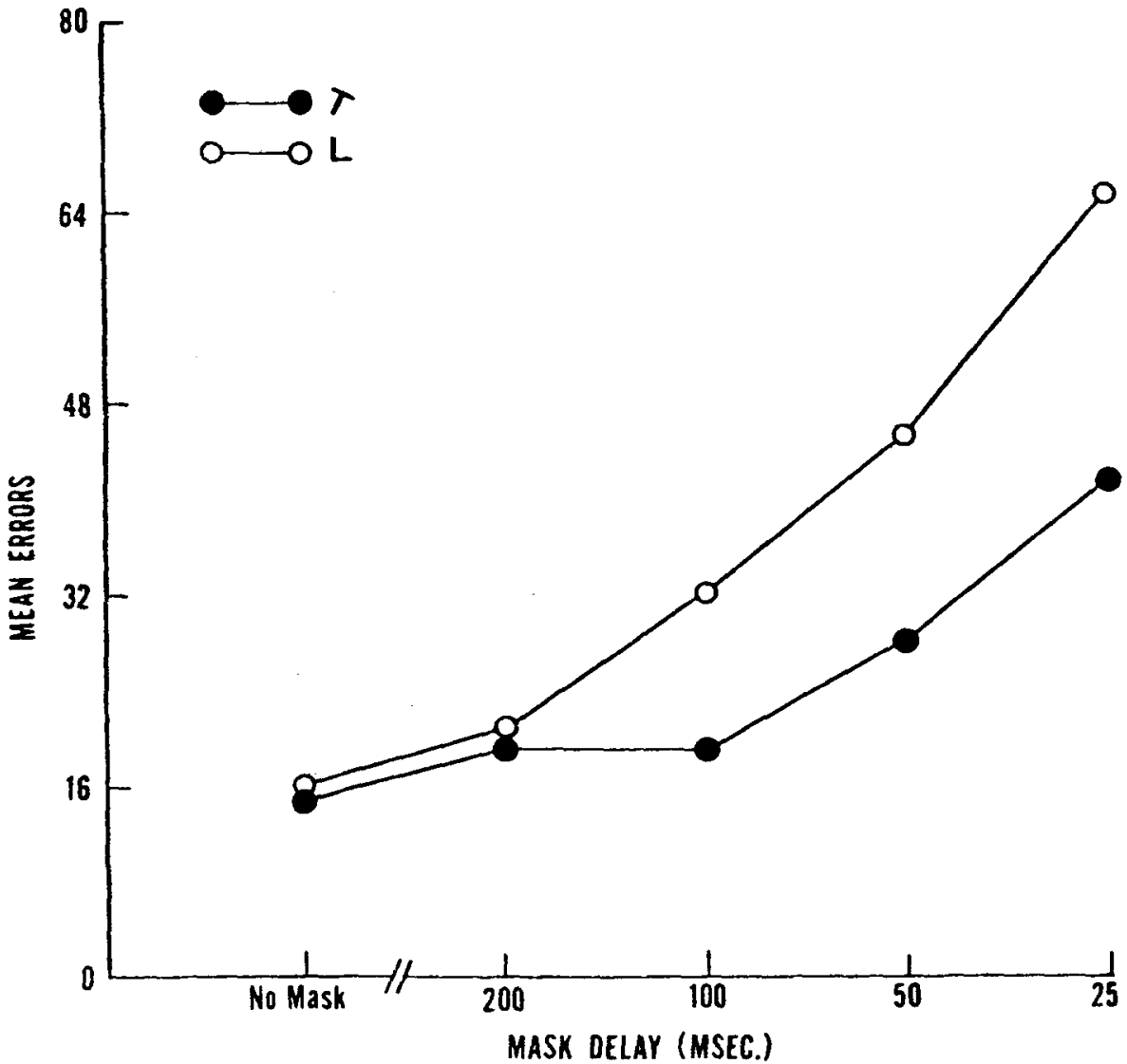

Fig. 2. Mean errors for a tilted $T$ and an $L$ as a function of mask delay in Experiment 1. The points are based on 1,536 trials (8 Ss $\times 192$ trials).

decreased by reducing the exposure duration. Reducing the exposure duration may be expected to reduce the adequacy of a stimulus but does not appear to change the time available for the inspection of the fading visual trace. Haber and Standing (1969) found that visual persistence was independent of stimulus duration over a range of 4 to $200 \mathrm{msec}$. Reducing the exposure duration should, therefore, decrease the discriminability of both the $L$ and the tilted $T$ figures, due to the decreasing clarity of the visual trace, but the discriminability of the tilted $T$ should not improve relative to that of the $L$. The experiment thus provides a control for the interpretation of the data offered in Experiment 1.

\section{Subjects}

Five volunteers with normal vision without correction served as paid Ss. They were naive as to the purpose of the experiment.

\section{Method}

The apparatus and stimuli were the s ame as those employed in Experiment 1, with the modification that no masking field was presented.
The stimulus array was presented at three durations of exposure: 55,35 , and $25 \mathrm{msec}$. These exposure durations were the same for all $S$ s in all experimental sessions. The procedure was the same in terms of practice and experimental sessions as that in Experiment 1, with the exception that only three experimental conditions were involved. Each $S$ was run for 5 days, with the 1st day a practice session. On each of the 4 experimental days, three blocks of 48 trials (one block for each of the three exposure durations) were presented with the tilted $T$ as the disparate figure and three blocks of 48 trials with the $L$ as the disparate figure.

\section{Results}

Figure 3 presents the mean errors for each of the three exposure durations. An analysis of variance indicated that only the increase in errors with decreasing exposure duration was significant $[F(2,8)=63.45, p<.01]$. Neither the figure type (whether the disparate letter was tilted $T$ or $L$ ) $[F(1,4)=3.34, p>.05]$ nor the Figure by Exposure Duration 


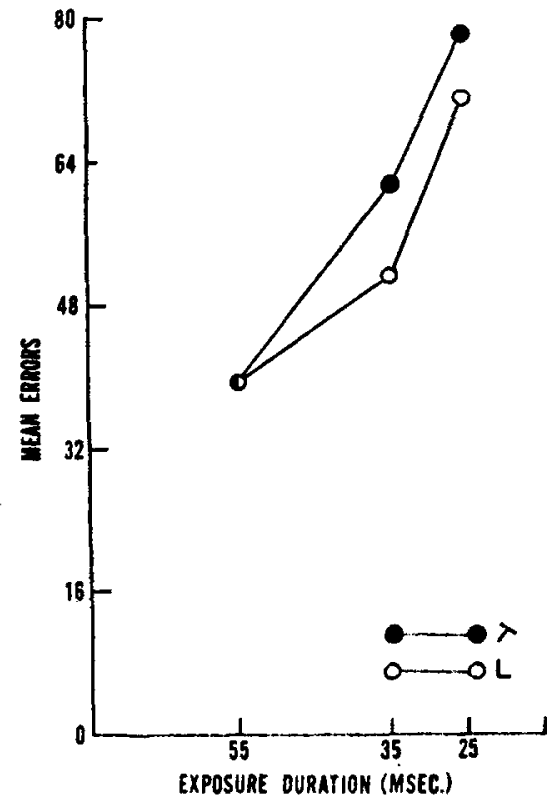

Fig. 3. Mean errors for a tilted $\mathbf{T}$ and an $L$ as a function of exposure duration in Experiment 2. The points are based on 960 trials (5 Ss x 192 trials).

interaction was significant $[F(2,8)=1.58, p>.05]$. It is clear that reducing discriminability of a tilted $T$ and an $L$ by reducing the exposure duration does not improve the discriminability of the tilted $T$ relative to that of the L. A signal detection analysis gave the same pattern of results. The results suggest that the decrease in stimulus duration produces a visual trace which is poorer in quality and which impairs the discriminability of a tilted $\mathrm{T}$ at least as much as that of an $L$. In contrast, reducing the delay of the masking field may be assumed to affect not only the quality of a visual trace but also the processes of analysis, i.e., whether concentrated attention may be brought to bear. The results thus support the hypothesis that the relatively better discriminability of the tilted $T$ in Experiment 1 is the consequence of the fact that a difference in line slope is more discriminable than a difference in the line arrangement when attention is distributed over a wide field.

\section{EXPERIMENT 3}

Experiment 3 examined the relative discriminability of a tilted $T$ and an $L$ in an empty visual field. The purpose of the experiment was to examine the relative discriminability of an $L$ and a tilted $T$ when a brightness difference between the figure and background directs a $S$ where to attend. Since the brightness difference is able to direct attention, the relative discriminability of an $\mathrm{L}$ and a tilted $\mathrm{T}$ should not be affected by reducing the delay between the offset of a stimulus array and the presentation of a masking pattern. Only with very short masking delays may the discriminability of a tilted $T$ be expected to improve relative to an $L$. The concentration of attention is not instantaneous and, with very short masking delays between the offset of a stimulus array and the onset of a masking pattern, the discriminability of a tilted $T$ should improve relative to an $\mathrm{L}$.

\section{Subjects}

Five volunteers with normal vision without correction served as paid Ss. They were naive as to the purpose of the experiment.

\section{Method}

The apparatus, stimulus arrangement, and procedure were the same as those in Experiment 1, except that only a single letter was presented in any of the six positions around the imaginary circle. An $S$ had to decide whether the stimulus was an upright $T$

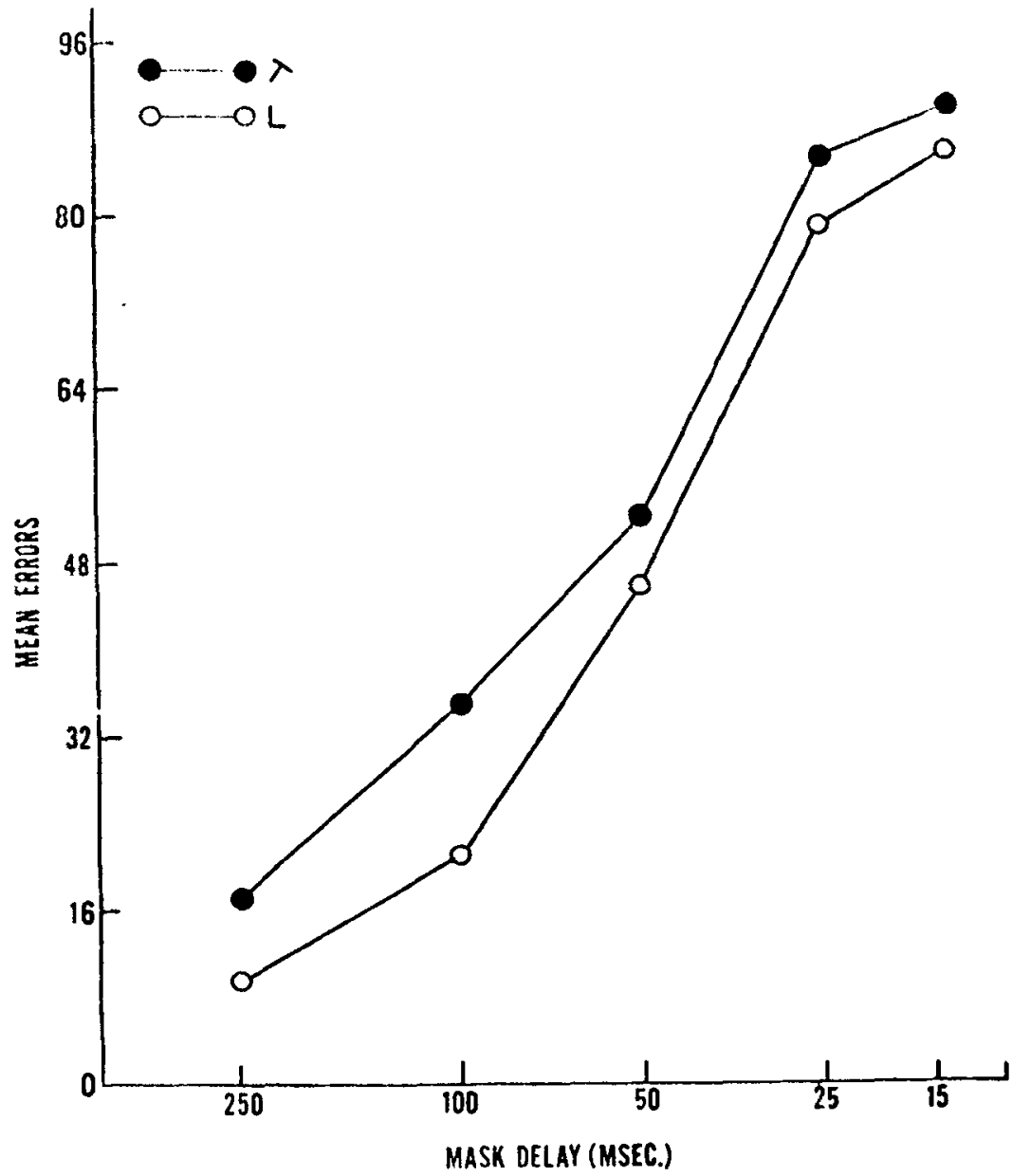

Fig. 4. Mean errors for a tilted $T$ and an $L$ as a function of mask delay in Experiment 3. The points are based on 960 trials (5 Ss x 192 trials). or a $\mathbf{T}$ tilted $33 \mathrm{deg}$ from the vertical, or an upright $T$ or an $L$ when a single figure could appear in any of six different possible positions. The letters were briefly flashed and were followed the letters had occupied. The five masking delays were: $250,100,50$, 25 , and 15 msec. Each $S$ was run for 5 days, with the first day as a practice session. On each of the 4 experimental block for each of the five masking conditions) were presented with a tilted $\mathrm{T}$ to be discriminated and five blocks of 48 trials were presented with an $\mathrm{L}$ to be discriminated. During the practice day, the exposure duration that gave an error rate of approximately $10 \%$ with a 250 -msec masking delay was determined. The exposure duration selected for each $\mathbf{S}$ was checked on each of the experimental days. For three Ss, this exposure duration was used on all 4 experimental days. For two $\mathrm{Ss}$, a slightly different exposure duration was used on 1 of the 4 experimental days. The mean exposure duration was by a masking pattern in the area that days, five blocks of 48 trials (one 
25.5 msec, with a range of 20 to 30 msec.

Results

Figure 4 presents the mean errors for each of the masking delays. The discriminability of the tilted $T$ was worse than the discriminability of the $\mathrm{L}$ at all masking delays. At 15- and 25-msec masking delays, the mean number of errors approached the chance level for both the tilted $T$ and the $L$. Because of this, the variances of the error scores for these masking delays were greatly reduced and the conditions for an analysis of variance were not met. An analysis of variance of the first three masking delays revealed significant effects due to delay of the masking field $[F(2,8)=16.70, p<.01]$ and the figure type (whether the disparate figure was a tilted $T$ or an $L)[F(1,4)=$ $11.44, \mathrm{p}<.05]$. The relative discriminability of the tilted $T$ and of the $\mathrm{L}$ remained the same as the delay of the masking field was reduced. The Figure by Masking Delay interaction was not significant $[F(2,8)$ $=3.03, p>.05]$. A signal detection analysis, however, suggested that the discriminability of a tilted $\mathrm{T}$ improved relative to that of an $\mathrm{L}$ at the shorter masking delays. The $d^{\prime}$ values at masking delays of $250,100,50,25$, and $15 \mathrm{msec}$ when an $L$ was the disparate figure were $3.22,2.46,1.41$, $.46, .26$, and when a tilted $T$ was a disparate figure were $2.74,1.80,1.21$, .31 , and .15 .

\section{EXPERIMENT 4}

In Experiment 3, the figures presented at 25 - and $15-$ msec masking delays were hardly seen at all and Ss responded close to chance level. Experiment 4 was concerned with whether the discriminability of a tilted $T$ relative to that of an $L$ would be impaired at these short masking delays if an adequate stimulus were presented. The purpose of Experiment 4 was to examine the relative discriminability of a tilted $T$ and an $L$ from an upright $T$ when the exposure duration of the stimuli was increased, providing a visual trace of improved quality.

\section{Subjects}

Five volunteers with normal vision without correction served as paid Ss. They were naive as to the purpose of the experiment.

\section{Method}

The experimental arrangement and the procedure were the same as in Experiment 3 , except that the exposure durations of the letters were increased for masking delays below $250 \mathrm{msec}$. For the 250-msec masking

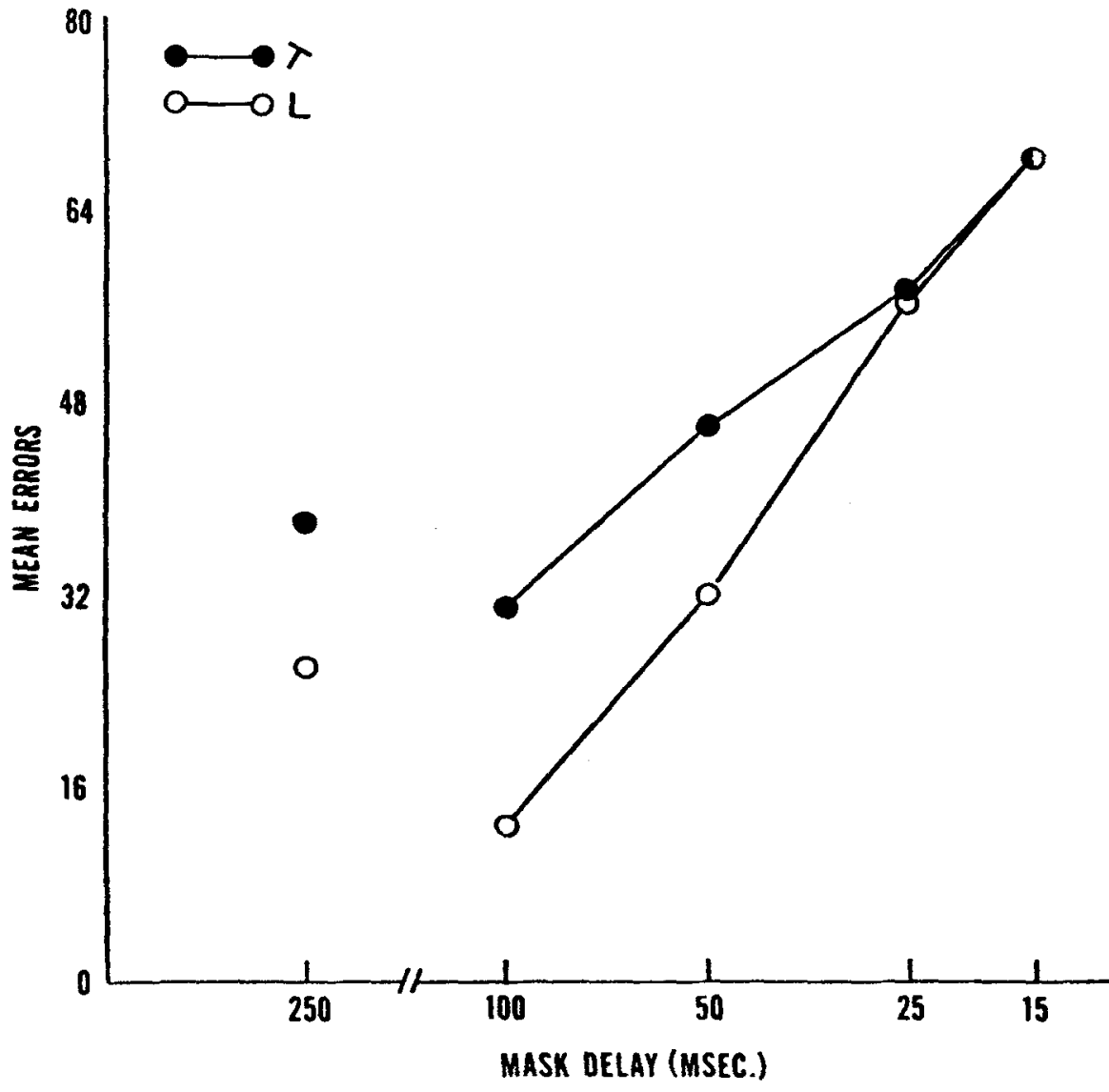

Fig. 5. Mean errors for a tilted $T$ and an $L$ as a function of mask delay in Experiment 4. The points are based on 960 trials (5 Ss $x 192$ trials). The stimuli at $250 \mathrm{msec}$ (unconnected points) were presented at a different exposure duration (see text).

delay, the exposure duration was kept at $25 \mathrm{msec}$. During the practice day, the exposure duration that gave an approximate error rate of between $10 \%$ and $20 \%$ with a masking delay of $100 \mathrm{msec}$ was determined. For one $S$, the exposure duration was the same on all 4 experimental days. For four $\mathrm{Ss}$, the exposure duration was slightly changed on either 1 or 2 of the experimental days. The mean exposure duration was $49.5 \mathrm{msec}$, with a range between 45 and 55 msec.

\section{Results}

Figure 5 presents the mean errors for each of the masking delays. The figure shows that the discriminability of a tilted $T$ was worse than that of the $\mathrm{L}$ for masking delays greater than 25 msec and that at masking delays of 25 and $15 \mathrm{msec}$ the mean errors for the tilted $T$ and the $\mathrm{L}$ became the same. At the 25-msec delay, the Ss were responding close to the threshold level, making a little more than $25 \%$ errors. An analysis of variance excluding the 250-msec masking delay revealed that the masking delay $[F(3,12)=62.16, p<.01]$ and the
Figure by Masking Delay interaction $[\mathrm{F}(3,12)=9.29, \quad \mathrm{p}<.01]$ were significant. The results thus indicate that in an empty visual field the discriminability of a tilted $T$ relative to that of an $L$ may improve with short masking delays. The $\mathrm{d}^{\prime}$ scores show the same pattern of results. An interpretation consistent with what we have proposed is that with short masking delays of $25 \mathrm{msec}$ and less, discriminability is based increasingly on differences that may be picked up when attention is distributed over a field.

An aspect of the results which should be noted is that in Experiment 4, when a single figure was presented, an $L$ tended to be discriminated better than a tilted $T$. In contrast, in Experiment 1, in a pattern of upright $\mathrm{Ts}$, the tilted $\mathrm{T}$ tended to be discriminated better than an $L$. A comparison of Figs. 2 and 5 shows the relative change in discriminability of the tilted $T$ and the $L$ figures. The exposure durations in Experiments 1 and 4 were comparable, and the results may be compared for the corresponding masking delays of 100 , 
50 , and $25 \mathrm{msec}$. The difference between the number of errors made by each $S$ at these masking delays with a tilted $T$ and an $L$ in Experiment 1 and in Experiment 4 was determined. The analysis of variance of these difference scores (unweighted-means solution for unequal $N$ ) revealed that the change in the relative discriminability of a tilted $T$ and of an $L$ in Experiments 1 and 4 was significant $[\mathrm{F}(1,11)=5.58$, $p<.05]$. The reason for the change in the relative discriminability of a tilted $T$ and of an $L$ is unclear. It is possible that the features of an $L$ in the visual trace are clearer than those of a tilted $T$ and that the discriminability of an $L$ is therefore better in an empty visual field. The vertical and horizontal lines of the upright Ts, however, would impair the discriminability of an $L$ in a pattern of upright Ts, while they might facilitate discriminating the diagonal lines of a tilted $\mathrm{T}$.

\section{DISCUSSION}

The present experiments indicate that discrimination of a difference in line slope is relatively better than discrimination of a difference in line arrangement when a mask makes a visual trace available for processing only for a short period of time. It is, perhaps, worth noting first that the results are consistent with the proposed explanation of similarity grouping as based upon the simultaneous pickup of stimulus differences in local spatial regions prior to a narrowing or focusing of attention (Beck, 1972). It is, of course, not intended to imply that the organization or grouping of features such as occur in the perception of a face may not have its basis in the symmetry, redundancy, or relationship of features to a schema. Rather, it is proposed that such grouping is based on subsequent encoding which differs from the primary segregation of the field into units.

Why does the discriminability of a tilted $T$ improve relative to that of an L when a mask limits the time available for processing items in a visual trace? Neisser (1967) has proposed two stages of processing: a preattentive parallel stage, which responds selectively to stimulus variables to which the visual system is highly sensitive, and a focal attentive process, which produces an identification of a stimulus based on both a more detailed analysis and memory. It is possible that the greater discriminability of a tilted $T$ is the result of the sensitivity of the preattentive process to differences in the slopes of lines. Information about the slopes of lines may be available to the preattentive process, while information about the arrangement of lines may require focal processing. One should be cautious, however, in assuming two separate processes. Another explanation is in terms of the attentional focusing process studied by Eriksen and Collins (1969; Eriksen \& Rohrbaugh, 1970). It is possible that the discrimination of a difference in the slopes of the component lines of a figure requires less of a narrowing of attention than the discrimination of a difference in the arrangement of the component lines of a figure. The critical question is, what kinds of information can be picked up in the periphery with distributed attention? It is possible that under distributed attention in peripheral vision, the visual system is more sensitive to simple variables like line slope than to relational variables like line arrangement. An $S$ begins with his attention distributed over the entire field. Thus, to discriminate between an upright $T$ and an $L$, the attentive field must be narrowed so that an $S$ attends to one or possibly two of the six figures in the array at a time. Because the clarity of the image decreases with time, an $\mathbf{S}$ is unable to inspect all six figures in an array. To pick up a tilted $T$, the attentive field may have to be narrowed to only three or four of the six figures in the array. The relatively better discrimination of a tilted $\mathbf{T}$ would be due to the greater number of figures which could be inspected before the array decayed beyond intelligibility. In an empty visual field, the brightness difference between a figure and the background attracts attention. The narrowing of attention, however, takes time, and the greater relative discriminability of a tilted $\mathrm{T}$ would be due to the fact that the discriminability of a difference in line slope can be picked up with a larger attentive field than can a difference in line arrangement.

\section{REFERENCES}

BECK, J. Effect of orientation and of shape similarity on perceptual grouping. Perception \& Psychophysics, 1966, 1, 300-302.

BECK, J. Perceptual grouping produced by line figures. Perception \& Psy chophysics. 1967, 2, 491-495.

BECK, J. Similarity grouping and peripheral discriminability under uncertainty. American Journal of Psychology, 1972, 85, 1-20.

ERIKSEN, C. W., \& COLLINS, J. F. Temporal course of selective attention. Journal of Experimental Psychology, $1969,80,254-261$.

ERIKSEN, C. W., \& ROHRBAUGH, J. W. Some factors determining efficiency of selective attention. American Journal of Psychology, 1970, 83, 330-342.

HABER, R. N., \& STANDING, L. G. Direct measures of short-term visual storage. Quarterly Journal of Experimental Psychology, 1969, 21, 43-54.

JULESZ, $B$. Visual pattern discrimination. IRE Transactions of the Professional Group on Information Theory, IT-8, $1962,84-92$.

NEISSER, U. Cognitive psychology. New York: Appleton-Century-Crofts, 1967.

OLSON, R. K., \& ATTNEAVE, F. What variables produce similarity-grouping? American Journal of Psychology, 1970, 83, 1-21.

\section{NOTE}

1. The number of errors due to a failure to respond were similar and less than $.1 \%$ in Experiment 1 when an $L$ was presented as the disparate figure and when a tilted $T$ was presented as the disparate figure. The number of errors due to a failure to respond was similar for an $L$ and a tilted $T$, and the total was less than $1 \%$ in each of the remaining experiments reported. These errors are included in the error scores reported.

(Accepted for publication February 7 1972.) 